\title{
Accident alert using GPS technology \& automated traffic light control for ambulance
}

\author{
Dhanoj M ${ }^{1}$, Ashwin Prem², Bibi Mohan ${ }^{3}$, Ashwin Prem ${ }^{4}$ \\ Assistant Professor, EIE Dept, Vimal Jyothi Engineering College, Kannur ${ }^{1}$ \\ Third Year Student, EIE Dept, Vimal Jyothi Engineering College, Kannur ${ }^{2,3,4}$
}

\begin{abstract}
Nowadays the main cause of death due to road accidents is not because of the effect of the accident on the person but because of the lack of care and sudden action after the event. This paper is meant to overcome this problem. Accident detector is a device which is meant to detect the presence of accident or shake over a limit that may occur to a vehicle during its motion. The system used in this paper is mainly a micro controller based system in which the functioning of all the components is controlled by a microcontroller. Here an accelerometer sensors the change in acceleration caused by the shake or tilt. The detection of accident is done by the microcontroller after checking the output of accelerometer. When the accident is detected, the location is decoded from the output of a GPS module and this data is send as a message to an emergency call center by using a GSM module.

The tremendous rise in number of vehicles that appear on the roads have increased so much that it creates blocks and chaos everywhere. The ambulances running on emergency could not always make it in time due to inappropriate traffic conditions and signals. The paper is meant to overcome this problem. The ambulance is provided with an RF transmitter which continuously transmits RF signals. Once the ambulance approaches the junction, the receiver which is placed some 50 meters away from traffic signal post captures the RF signals which are transmitted by the transmitter. Upon receiving signals, the traffic signals are controlled for the unhindered passage of ambulance. The light goes green to all directions to the easy flow of traffic in the lane where the ambulance is. The light at all other lane will go red to avoid traffic blocks. This control sustains for a predetermined period until the receiver is in the range of signal availability. After the ambulance passes, the traffic control then fails to the normal sequential flow.
\end{abstract}

Keywords: GSM module, RF signals, GPS module.

\section{INTRODUCTION}

As a primary result of population explosion the number of individuals using automobiles is increasing. In the modern world the rate of accidents are increasing day by day. Most of the accident deaths are caused due to ignorance and lack of sufficient remedial measures and timely action. All these facts points to an efficient accident monitoring and vehicle tracking system.

In this paper, we are trying to implement a system by which, we can effectively tackle this situation by introducing an accident detection and vehicle tracking system. Simply the system works on the basic principle by which the variation of acceleration of the vehicle due to accident is detected. With the help of special devices accident is detected and message is send towards responsible authorities and proper action can be taken by them. So in this way through this paper we can implement an efficient accident alert system.

By implementing this system we are hoping to have better control over road accidents by the fast communication between the victim and the control centre. In this way we can minimise the possibility of accidental death. So we hope that the project will in fact open away to a better control of the traffic. The system in the simple way can be explained by connecting the components involved in it. The primary and foremost component that detect the occurrence of the accident is the accelerometer. A microcontroller ATmega328 controls the working of the whole components in the system. The output of the accelerometer operates an alarm for a particular time interval as provided in the program.

After this time interval the message will be send to the control centre using a GSM module and the message is decoded and the correct location of the vehicle is located using GPS system with the help of Google map. In today's world health hazards are a major concern. Especially people in the older age group are the victims, and moreover the traffic conditions are worsening day by day, which results in traffic jams.

Many important jobs get delayed due to these traffic jams. Ambulance service is one of the major services which get affected by traffic jams. To solve this problem we have come up with the solution of "intelligent ambulance with automatic traffic control".

Traffic in a city is very much affected by traffic light controllers. Waiting for the light to change into appropriate signals could end up in loss of life in case of emergency vehicles.

Ambulances running on emergency could not afford for the light gone red on traffic islands. Using algorithms traffic can be controlled much more efficiently. Time and life could be saved hence to an extent on road. 
II. BLOCK DIAGRAM

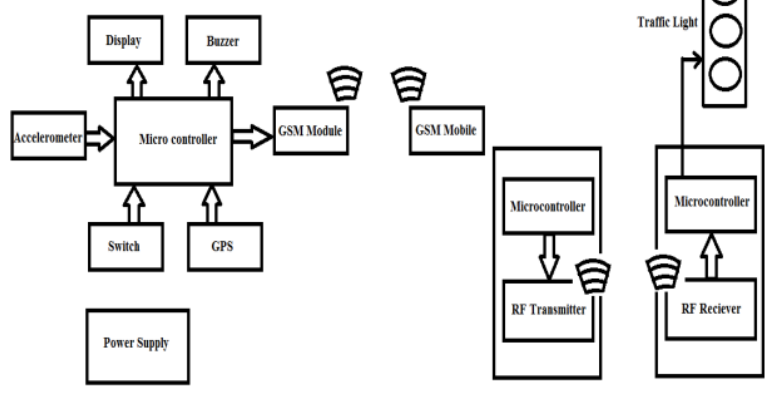

As shown in the diagram the main components of the system are a microcontroller, an accelerometer, a GPS module, Microcontroller, RF transmitter, RF receiver, power supply and a GSM. Accelerometer is meant for the detection of change in acceleration, GPS module is for finding the co-ordinates of the location, GSM for sending message, microcontroller controls all these operations. Accelerometer detects the change in acceleration of the moving mass. The output of the accelerometer will be an analog quantity which is proportional to the change in acceleration. The output of the accelerometer is given to the microcontroller as input. The microcontroller compares the value of input with the programmed value. A value more than the programmed value indicates that an accident is detected. At the time when the accident is detected, the alarm circuit will start working by a beep sound. The sound lasts for about 20 seconds. If the accident is not severe the driver himself can stop the further action by simply pressing a switch attached to the controller.

In the case of a severe accident the driver may not be able to control the further action. During this situation, the controller extracts the output of the GPS. The output of the GPS will be a large string consisting of a wide range of data about the position. From this data controller select the required data (latitude and longitude) for the purpose of the position detection. Using a GSM module the extracted data is send to an emergency call centre as an sms. The GSM module in the receiver station receives the message which in turn is connected to a personal computer where the message is decoded and exact location is found out with the help of the Google map. Now the RF transmitter is meant for transmitting continuously .

RF receiver captures RF signals transmitted by the transmitter placed on the ambulance. Two microcontrollers has been used here, one of them has been placed in the ambulance interfaced with the transmitter and the other one is placed interfaced with the receiver. The later one controls the traffic light at the junction. Once the ambulance approaches the junction, the receiver captures the signal transmitted by the transmitter as it comes into the suitable range of operation. Upon receiving signals the controller controls the traffic lights in convenience of the ambulance. After the ambulance closes the junction, the traffic comes back to normal flow.

\section{GPS MODULE}

GPS or Global Positioning System mainly consists of an array of satellites orbiting the earth at a particular orbit. Basically it is a space based satellite navigation system, which provides the exact location and time of the particular object on the surface of the earth. GPS system works by sending and receiving signal in the form of radio waves from the earth and to the earth. GPS satellites are orbiting the earth an altitude of 11,000 miles. It uses radio waves for the communication because radio waves travel at speed of 186,000 miles/sec. Here in this project we are using ITead studio GPS module version 1.1. The main features of the module are given below.

- The system is having a 20 Channel receiver.

\section{$\bullet$}

It is having extremely high sensitivity.

High accuracy

It uses a current and voltage of $70 \mathrm{~mA}$ at $4.5-6.5$

\section{volt}

- Weight-16 grange

Size $30 \mathrm{~mm} * 30 \mathrm{~mm} * 10.5 \mathrm{~mm}$

Built in patch antennae

Extremely fast TTFF (Time To First Fix)

While installing GPS shield to Arduino, we have to check the operation voltage level of development board. If the voltage is $3.3 \mathrm{~V}$ (IFLAT32, Leaf maple), we have to set the Operation Level Setting switch to 3.3V. If the voltage is $5 \mathrm{~V}$ (Arduino), we have to set the Operation Level Setting switch to $5 \mathrm{~V}$. The system consist of three parts

a) 24-32 Satellites (only twelve are visible at once) in orbit around the earth.

b) Ground station for monitoring and controlling the satellites.

c) A GPS receiver that calculates the position.

The twenty-four satellites are in a certain orbit around our planet. They continuously broadcast a signal down to earth including the current time and orbit information. The math and equations for determining a precise position is extremely complex, but the general concept is simple.

It takes four satellites to determine your position. Each of these satellites transmits a time signal. By comparing the time sent from the satellite to the GPS receiver's own clock, the elapsed time of the transmission can be determined. Because the position of the satellite is known and because we know how fast the message travels through space, the distance between the receiver and the satellite can be determined. The receiver then repeats this with the other three satellites and triangulates a position.

There are three ways a GPS coordinates are expressed. One is decimal (looks like $83.5672 \mathrm{~N} 48.4532 \mathrm{~W}$ ) this one is easy for computers to understand. The second is degreeminutes-seconds, which looks like 38 $53^{\prime} 23^{\prime \prime} \mathrm{N}$, $77^{\circ} 00^{\prime} 32^{\prime} \mathrm{W}$. The last is degree minutes.

If the GPS says it is at $4008.5398 \mathrm{~N} 08304.7582 \mathrm{~W}$, then it is really $\mathrm{N} 40^{\circ} 08.5398^{\prime} \mathrm{W} 83^{\circ} 04.7582^{\prime}$. Just add a degree sign two digits before the decimal point.

Google Earth and Google Maps will not display the raw GPS output coordinate. We must convert it into degreeminutes. 


\section{GSM MODULE}

As known by all the GSM represents the Global System for Mobile communication. It is provided with special slots for accepting SIM card through which the message is send. The receiver section consists of a quad band GSM receiver which is controlled by AT commands. Depending up on the transmitting power GSM can be classified into 2 classes that is class 1 and class 4 . Class 1 requires $2 \mathrm{~W}$ power for transmission. We can use SIM 1900 GSM module. A GSM network is composed of several functional entities, whose functions and interfaces are specified.

The GSM network can be divided into three broad parts, the Mobile Station is carried by the subscriber. The Base Station Substation controls the radio link with Mobile Station. The Network Subsystem, the main part of which is the Mobile service Switching Centre (MSC), performs the switching of calls between the mobile users, and between mobile and fixed network users. The MSC also handles the mobility management operations. Not shown is the Operations and Maintenance Centre, which oversees the proper operation and setup of the network. The Mobile Station and the Base Station Subsystem communicate across the Um interface, also known as the air interface or radio link. The Base Station Subsystem communicates with the Mobile services Switching Centre across the A interface. The mobile equipment is uniquely identified by the International Mobile Equipment Identity (IMEI). The SIM card contains the International Mobile Subscriber Identity (IMSI) used to identify the subscriber to the system, a secret key for authentication, and other information. The IMEI and the IMSI are independent, thereby allowing personal mobility. The SIM card may be protected against unauthorized use by a password or personal identity number. The Base Station Subsystem is composed of two parts, the Base Transceiver Station (BTS) and the Base Station Controller (BSC). These communicate across the standardised Abis interface, allowing (as in the rest of the system) operation between components made by the different suppliers.

\section{SIM900}

SIM900 is a quad-band GSM/GPRS engine that works on frequencies GSM 850Mhz, EGSM 900Mhz, DCS $1800 \mathrm{Mhz}$ and PCS 1900Mhz. SIM900 features GPRS multi-slot class 10/class 8 (optional) and supports the GPRS coding schemes CS- 1 , CS-2, CS-3 and CS-4. With a tiny configuration of $24 \mathrm{~mm} \times 24 \mathrm{~mm} \times 3 \mathrm{~mm}$, SIM900 can meet almost all the space requirements in your applications, such as M2M, smart phone, PDA and the other mobile devices. The physical interface to the mobile application is a 68-pin SMT pad, which provides all hardware interfaces between the module and customers' boards.

Features

The keypad and SPI display interface will give you the flexibility to develop customised applications 8

- $\quad$ Serial port and Debug port can help you easily develop your applications.
- $\quad$ One audio channel includes a microphone input and a speaker output. The SIM900 is designed with power saving technique so that the current consumption is as low as $1.5 \mathrm{~mA}$ in sleep mode.

- $\quad$ Power supply: Single supply voltage $3.4 \mathrm{~V}-4.5 \mathrm{~V}$ firmware

- Low power consumption

The Sim900 is integrated with the TCP/IP protocol; extended TCP/IP AT command are developed for customers to use the TCP/IP protocol easily, which is very useful for those data transfer applications

\section{ADVANTAGES}

Efficient monitoring of accident

Fast communication between the victim and the control station

\section{- $\quad$ Fast tracking of vehicle \\ Accuracy in finding in location \\ Ease of movement of Ambulance in traffic}

\section{CONCLUSION}

We conclude the paper by believing that the project was successful up to a level as we are concerned. We are very much happy that the hard work that we had put into this paper was fruitful. All the components individually and collectively responded to the condition what we had given in the program. Accelerometer responded towards the change in acceleration when it was given a particular amount of shake and through GPS module we were able to find the latitude and longitude of that particular location. So by looking at the overall performance we are now very much confident enough to completely implement an efficient accident detection system in future.

The ambulance is provided with an RF transmitter which continuously transmits RF signals. Once the ambulance approaches the junction, the receiver which is placed some 50 meters away from traffic signal post captures the RF signals which are transmitted by the transmitter. Upon receiving signals, the traffic signals are controlled for the unhindered passage of ambulance. The light goes green to all directions for the easy flow of traffic in the lane where the ambulance is. The light at all other lane go red to avoid traffic blocks.

This controls sustains for a predetermined period until the receiver is in the range of signal availability. After the ambulance passes the traffic control then falls to normal sequential flow.

\section{REFERENCES}

http://arduino.cc/en/Reference/SoftwareSerial http://emguide.wordpress.com/2012/12/10/15

http://www.arduino.cc/guide/introduction

http://en.wikipedia.org/wiki/rf_module

http://en.wikipedia.org/wiki/arduino

http://www.engineersgarage.com

http://www.sparkfun.com/datasheets

www.arduino.cc

www.probots.co.in

www.iteadstudio.com

www.sparkfun.com/datasheets 